\title{
Altered Acquisition and Extinction of Amphetamine-Paired Context Conditioning in Genetic Mouse Models of Altered NMDA Receptor Function
}

\author{
Michael A Benneyworth' and Joseph T Coyle*,' \\ 'Department of Psychiatry, Harvard Medical School, McLean Hospital, Belmont, MA, USA
}

\begin{abstract}
Repeated intermittent exposure to amphetamine (AMPH) results in the development of persistent behavioral and neurological changes. When drug exposure is paired with a specific environment, contextual cues can control conditioned responses, context-specific sensitization, and alterations in dendritic morphology in the nucleus accumbens (NAc). Intact N-methyl-D-aspartate (NMDA) glutamate receptor signaling is thought to be required for associative learning. The acquisition of context-specific behavioral sensitization to $\mathrm{AMPH}$ and extinction of conditioned hyperactivity have been investigated in two genetically modified mouse strains: the serine racemase homozygous knockout (SR-/-) and glycine transporter I heterozygous mutant (Gly $\mathrm{TI}-/+)$. These strains have reciprocally altered NMDA receptor co-agonists, D-serine and glycine, levels that result in decreased (SR-/-) or increased (GlyTI - / +) NMDA receptor signaling. AMPH-induced changes in dendritic morphology in the NAc were also examined. SR-/- mice showed reduced expression of context-specific sensitization and conditioned hyperactivity. However, the conditioned hyperactivity in these mice is completely resistant to extinction. Extinction reversed AMPH-induced increased in NAc spine density in wild-type but not SR-/- mice. GlyTI -/+ mice showed a more rapid acquisition of sensitization, but no alteration in the extinction of conditioned hyperactivity. The SR-/- data demonstrate that a genetic model of NMDA receptor hypofunction displays a reduced ability to extinguish conditioned responses to drug-associated stimuli. Findings also demonstrate that the morphological changes in the NAc encode conditioned responses that are sensitive to extinction and reduced NMDA receptor activity. NMDA receptor hypofunction may contribute to the comorbidity of substance abuse in schizophrenia.
\end{abstract}

Neuropsychopharmacology (2012) 37, 2496-2504; doi:I0.1038/npp.20I2.108; published online 4 July 2012

Keywords: NMDA receptor; D-serine; glycine; addiction; behavioral sensitization; extinction

\section{INTRODUCTION}

Repeated intermittent psychostimulant exposure results in behavioral sensitization, often measured as an increased efficacy of that drug to stimulate locomotor activity (Vezina and Leyton, 2009). The acquisition of sensitization is facilitated by associative learning that links a specific context to drug exposure (Badiani et al, 1995). Specific alterations in immediate early gene expression and neuronal morphology are observed following such contextspecific sensitization (Robinson and Kolb, 1997; Mattson et al, 2008; Koya et al, 2009; Singer et al, 2009). Increased dendritic branching and spine density of medium spiny neurons in the nucleus accumbens (NAc) occur only with

*Correspondence: Dr JT Coyle, Department of Psychiatry, Harvard Medical School, McLean Hospital, I 5 Mill Street, MRC 122, Belmont, MA 02478, USA, Tel: + I 617855 2101, Fax: + I 6178552705 ,

E-mail: Joseph_coyle@hms.harvard.edu

Received 20 December 2011; revised I4 May 2012; accepted 31 May 2012 context-dependent sensitization but not following a context-independent paradigm (Singer et al, 2009). This observation supports the hypothesis that these changes are part of the associative learning. When inducing contextdependent sensitization, a concomitant conditioned hyperactivity develops (Anagnostaras and Robinson, 1996). This conditioned response (CR) is observed in the presence of the conditioned stimulus (CS; drug-paired environment) without drug exposure and represents another measure of associative learning that may be encoded by the neurological changes.

Excitatory neurotransmission mediated by the $\mathrm{N}$-methylD-aspartate (NMDA) subtype of glutamate receptors plays a critical role in synaptic plasticity and memory formation (Tsien, 2000). NMDA receptors are heterotetrameric ionotropic receptors comprising NR1 and NR2 subunits. These functionally complex receptors require agonist binding at both the glutamate site on NR2 subunit and the glycine modulatory site (GMS) on NR1 subunit. NMDA receptor activation leads to a dramatic influx of $\mathrm{Ca}^{2+}$, which then triggers intracellular signaling cascades that are 
involved in restructuring the postsynaptic membrane in accordance with various forms of synaptic plasticity (Bear and Malenka, 1994; Malenka and Nicoll, 1999). Selective ablation of NR1 expression in striatal neurons has been used to study the role of NMDA receptor signaling in the acquisition of behavioral sensitization and conditioned place preference (Heusner and Palmiter, 2005; Agatsuma et al, 2010). These studies clarify previous research showing that blockade of NMDA receptor is disruptive to the development of sensitization and place preference (Haracz et al, 1995; Kim et al, 1996; Li and Wolf, 1999).

In addition to its role in the acquisition of conditioned behavior, recent research has begun to illustrate the importance of NMDA receptor signaling to the reversal or extinction of conditioned behaviors. Specifically, potentiation of NMDA receptor neurotransmission, with the GMS partial agonist $\mathrm{D}$-cycloserine, has been used as a strategy to facilitate the extinction of place conditioning (Paolone et al, 2009; Thanos et al, 2009; Myers and Carlezon, 2010). These findings point to the potential therapeutic benefit of enhancing NMDA receptor signaling, but questions remain as to whether aberrant NMDA receptor function may contribute to genetic susceptibility to addiction.

Glycine and D-serine are the endogenous agonists of the NMDA receptor GMS. Synaptic availability of glycine in the brain is regulated by the glycine transporter subtype 1 (GlyT1). D-serine is synthesized through the racemization of its L-isomer by the enzyme serine racemase (SR). The understanding of the functional impact of GlyT1 and SR has been advanced by the use of recombinant mouse lines bearing knockout mutations to the genes encoding these proteins (Tsai et al, 2004; Martina et al, 2005; Basu et al, 2009; Devito et al, 2010; Balu et al, 2012). The SR homozygous knockout (SR-I-) mutant and GlyT1 hypomorph (GlyT1-/+) represent functionally converse mutations that lead to either NMDA receptor hypofunction (SR-I-; Basu et al, 2009) or hyperfunction (GlyT1-/+; Martina et al, 2005). Behavioral sensitization and conditioned hyperactivity were tested in these mouse strains to investigate the hypothesis that NMDA receptor signaling plays a critical role in the acquisition and extinction of behavior driven by contextual conditioning. Further analysis of neuronal architecture was used to investigate the hypothesis that dendritic spine density changes within the NAc may represent a reversible alteration that is specifically related to conditioned responses that are expressed in the absence of drug.

\section{MATERIALS AND METHODS}

\section{Subjects}

Wild-type (WT) C57BL/6J mice derived from in-house breeding were used for WT-only experiments in these studies and were no more than one generation removed from mice imported from the Jackson Laboratory (Bar Harbor, ME). The SR mutant construct consists of a targeted deletion of exon 1, which encodes the catalytic domain of the enzyme (Basu et al, 2009). Experimental subjects were derived from SR heterozygote $(-I+)$ crosses to produce SRnull mutant $(-/-)$ and WT littermate controls. The GlyT1 mutant construct is a targeted deletion of exons 2 and 3 (Tsai et al, 2004). Both constructs were backcrossed onto a
C57BL/6J background for $>10$ generations. Because of the lethality of the homozygous GlyT1-null mutants, experiments were conducted using heterozygote mutant $(-l+)$ mice. Experimental mice were derived from GlyT1-/+ crosses to WT mice. Experimental subjects were housed four per cage in a vivarium on a 12/12 h light/dark cycle with food and water provided ad libitum. A total of 192 animals (age 9-15 weeks of age) were used for these studies (116 WT, 51 SR $-/-$, and 25 GlyT1-/+). All subjects were habituated to experimenter handling and injections before behavioral testing. The principals of laboratory animal care were followed in accordance with the 'Guidelines for the Care and Use of Mammals in Neuroscience and Behavioral Research' (National Research Council 2003).

\section{Behavioral Sensitization and Conditioned Hyperactivity}

Apparatus. Activity was monitored using open field chambers $(27 \times 27 \times 20 \mathrm{~cm}$; ENV-510, Med Associates, St Albans, VT) equipped with 16 horizontal ( $x$ - and $y$-axes) and 16 vertical ( $z$-axis) infrared photobeams. Chambers were contained in sound-attenuating cubicles, illuminated by two 28V, 0.1A lights. Photobeam breaks were recorded and converted to distance traveled using activity-monitoring software (Activity Monitor 5.0; Med Associates). The threshold for detection of ambulation was set at 3 consecutive beam breaks in $500 \mathrm{~ms}$. Test sessions were $60 \mathrm{~min}$ in length, with data collected in 5 min bins.

Experimental conditions. A schematic of the drug treatment and testing schedule used to induce behavioral sensitization and conditioned hyperactivity is shown in Figure 1a. The design was used to perform four experiments: (1) acquisition of behavioral sensitization and extinction of conditioned hyperactivity in WT mice; (2) characterization of conditioned hyperactivity in WT mice; (3) SR $-/-v s$ WT littermates; and (4) GlyT1-I+ vs WT littermates. Subjects were habituated to the test chambers in a 60 -min session 3 days before the start of sensitization. Subjects received a 5day treatment regimen of amphetamine (AMPH; $3.0 \mathrm{mg} / \mathrm{kg}$ for experiments 1,2 , and $3 ; 2.0 \mathrm{mg} / \mathrm{kg}$ for experiment 4 ) or saline. Locomotor activity was determined for the $60 \mathrm{~min}$ immediately following injection to assess the acquisition of sensitization. At 14 days after the final treatment, all mice were challenged with a single dose of AMPH $(3.0 \mathrm{mg} / \mathrm{kg}$ for experiments 1,2 , and 3 , or $2.0 \mathrm{mg} / \mathrm{kg}$ for experiment 4 ) to assess the expression of sensitization. Conditioned hyperactivity was observed after saline injection starting 3 days after the challenge test. Extinction of conditioned hyperactivity was achieved by repeating this test, that is, exposure to the test chamber in the continued absence of AMPH exposure. All injections were s.c.

\section{Golgi Staining and Analysis of Dendritic Spine Density}

Golgi staining was performed as previously described (Balu et al, 2012) with a few modifications. Whole brains from subjects used in behavioral tests ( $n=6-7 /$ group) were rapidly removed from the skull and fixed for $64 \mathrm{~h}$ using the Rapid GolgiStain Kit (FD NeuroTechnologies, Ellicot City, MD) according to the manufacturer's instructions. The fixed brains were sliced on a freezing microtome at a 
a

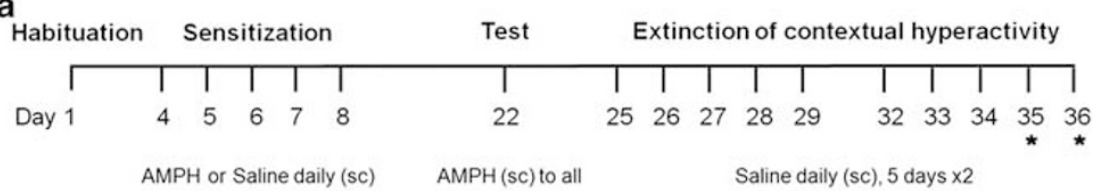
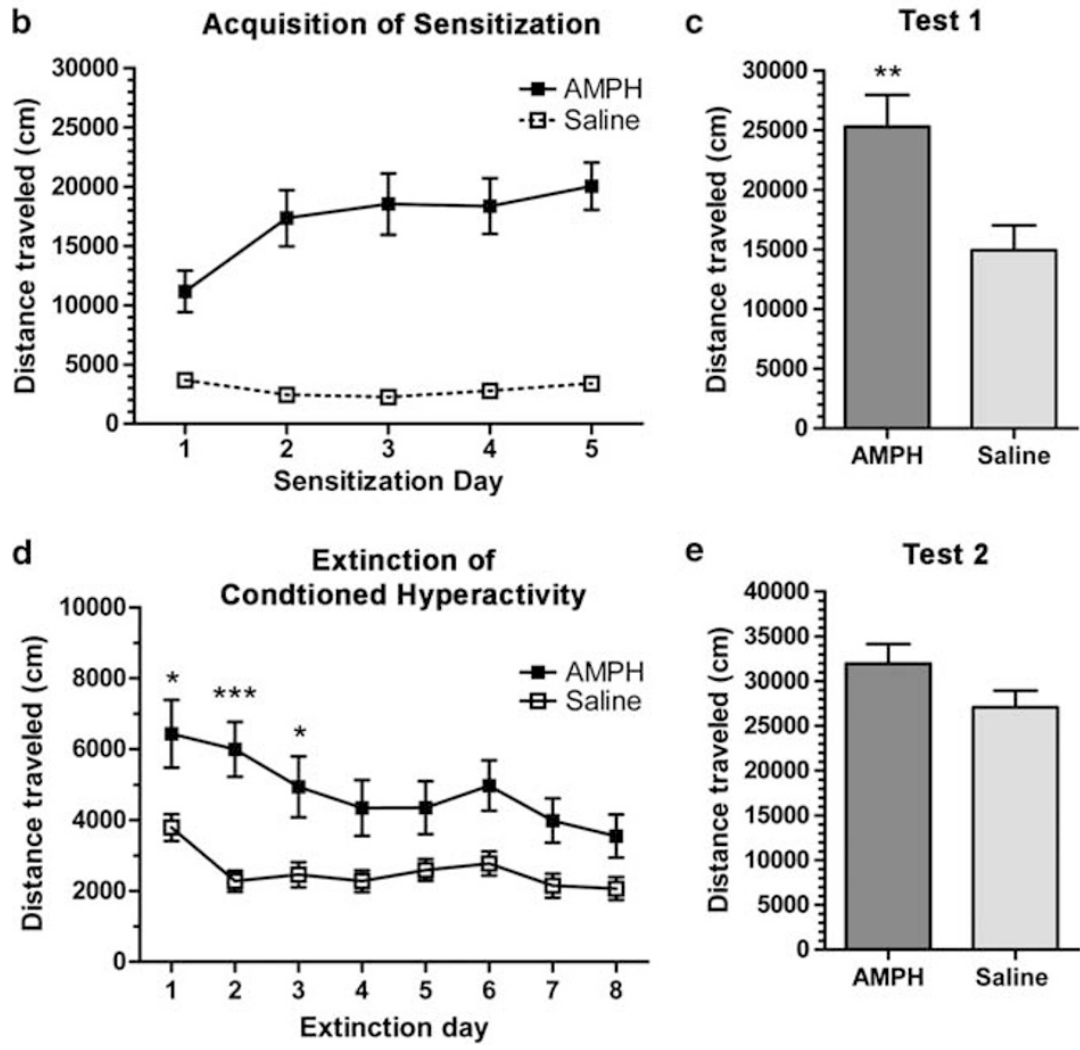

Figure I Acquisition of context-specific sensitization and extinction of conditioned hyperactivity. Injection schedule is shown in (a). Wild-type mice $(n=10-1 \mathrm{I})$ received injections of saline (open squares) or AMPH $(3.0 \mathrm{mg} / \mathrm{kg}$, filled squares) immediately before activity monitoring for 5 days to induce context-specific sensitization. A challenge test (AMPH, $3.0 \mathrm{mg} / \mathrm{kg}$ ) was performed 14 days later. After days of the challenge test, conditioned hyperactivity was observed over the course of 8 extinction sessions (* indicates sessions that were subsequently added for SR-1- studies). The acquisition of context-specific sensitization is shown in (b) and the first challenge test in (c) $(* * *<0.01$, Student's $t$-test, compared with saline pre-exposed control). Extinction of conditioned hyperactivity is shown in (d). Mice sensitized to AMPH show increased locomotion following saline treatment than the saline pre-exposed controls $(* p<0.05$, $* * * *<0.00 I$, Bonferroni). At 5 days after extinction, mice were challenged again with AMPH as shown in (e). There was no significant expression of sensitization; however, all groups were elevated as compared with challenge test I. Results are presented as means \pm SEM.

thickness of $120 \mu \mathrm{m}$. Sections were mounted on 3\% gelatincoated slides $(75 \times 25 \mathrm{~mm}$; FD NeuroTechnologies $)$. Sections were kept at room temperature overnight to dry, protected from light. Slides were then stained, dehydrated, and cleared with xylene according to the manufacturer's protocol. Medium spiny neurons in the NAc core (NAcc) were chosen for reconstruction. Four neurons were analyzed per hemisphere per subject. As previously described (Singer et al, 2009), the hemisphere was the unit of analysis, and only hemispheres containing 4 suitable neurons were included in the analysis, resulting in an $n=11-14$ /group. Two terminal dendrites $(40-150 \mu \mathrm{m}$ in length) per neuron were traced at $\times 40$ on a Zeiss Axioskop40 microscope, and spines were identified at $\times 100$ (oil-immersion). Dendrite length and spine density (spines $/ 10 \mu \mathrm{m}$ ) were calculated using Neurolucida (MBF Bioscience, Williston, VT).

\section{Statistics}

Total locomotion during the $60 \mathrm{~min}$ test sessions was summarized and analyzed for drug treatment-dependent and genotype-dependent effects on behavioral sensitization and conditioned hyperactivity. Two-way ANOVA with Bonferroni-corrected post hoc $t$-tests was used for statistical analysis of locomotion and spine density.

\section{RESULTS}

\section{Behavioral Sensitization and Conditioned Hyperactivity in WT Mice}

Before sensitization, locomotor activity was examined to assess baseline activity and also to habituate novelty-induced activity in the test chamber. Subjects ( $n=10-11 /$ group) 
were then assigned to either the AMPH $(3.0 \mathrm{mg} / \mathrm{kg}$, s.c.) or saline treatments, counterbalanced for baseline activity (4601 \pm 497 vs $4096 \pm 453$, respectively). Behavioral sensitization was induced by daily AMPH treatment (Figure 1). One-way ANOVA demonstrated a progressively increasing locomotor response to AMPH $(\mathrm{F}(4,10)=25.75, p<0.001$; Figure $1 \mathrm{~b}$ ) and Student's $t$-test analysis of test 1 showed an effect of previous AMPH exposure as compared with saline control subjects $(p<0.01$; Figure $1 c)$.

Following context-dependent sensitization, subjects were retested in the same test chambers for conditioned hyperactivity following saline treatment. Conditioned hyperactivity is observed as a greater degree of locomotion in the subjects sensitized to AMPH as compared with the saline-treated controls and was progressively extinguished by repeated context exposure in seven subsequent sessions (Figure 1d). Conditioned hyperactivity was revealed by twoway ANOVA illustrating a main effect of sensitizing AMPH exposure $(\mathrm{F}(1,133)=8.74, p<0.01)$ and Bonferroni post hoc $t$-test showed specific differences in activity between AMPHtreated and saline-treated control on extinction days 1-3 $\left({ }^{*} p<0.05,{ }^{* * *} p<0.001\right)$. The extinction of conditioned hyperactivity is shown by a significant interaction of AMPH exposure and extinction training $(\mathrm{F}(7,133)=2.74, p<0.05)$. No significant conditioned hyperactivity was observed in the AMPH-treated subjects on extinction days 4-8. At 5 days after the last extinction session, subjects were re-challenged with AMPH $(3.0 \mathrm{mg} / \mathrm{kg})$. AMPH response was elevated in all subjects, and there was no longer a statistical difference between treatment groups (31963 vs 27063, $t=1.69$, Student's $t$-test). This finding confirms that the sensitized response is still intact after extinction of conditioned hyperactivity and that a single AMPH injection (challenge test 1) produces significant long-lasting sensitization.

\section{Characteristics of Conditioned Hyperactivity in WT Mice}

In order to further define the characteristics of this conditioned hyperactivity, we looked at its context dependence and the dependence of the extinction learning upon re-exposure to that context. To test the context specificity, locomotor activity was compared between AMPH-sensitized and saline-treated controls $(n=10 /$ group $)$ in the test chamber on day 1 of the extinction training and a home cage-like environment on the following day (Figure 2a). Two-way ANOVA showed significant main effects of chamber type $(\mathrm{F}(1,18)=31.31, p<0.0001)$ and AMPH treatment $(\mathrm{F}(1,18)=7.45, p<0.05)$. The interaction was not significant $(\mathrm{F}(1,18)=3.10)$, likely owing to the large effect of the test chamber type. Activity was not different between treatment groups in the home cage-like chamber (8576 \pm 1074 vs $7967 \pm 815$ ), whereas these same subjects displayed a robust conditioned hyperactivity in the test chamber (5648 \pm 518 vs $2348 \pm 324, p<0.01$, Bonferroni). The same subjects were tested again in a second extinction session 10 days later to see whether the conditioned hyperactivity declined in the absence of re-exposure to either the conditioned or unconditioned stimuli (Figure 2b). AMPH-sensitized subjects exhibit a robust conditioned hyperactivity, as compared with saline controls, in the second extinction session $(4706 \pm 594$ vs $1568 \pm 297$,
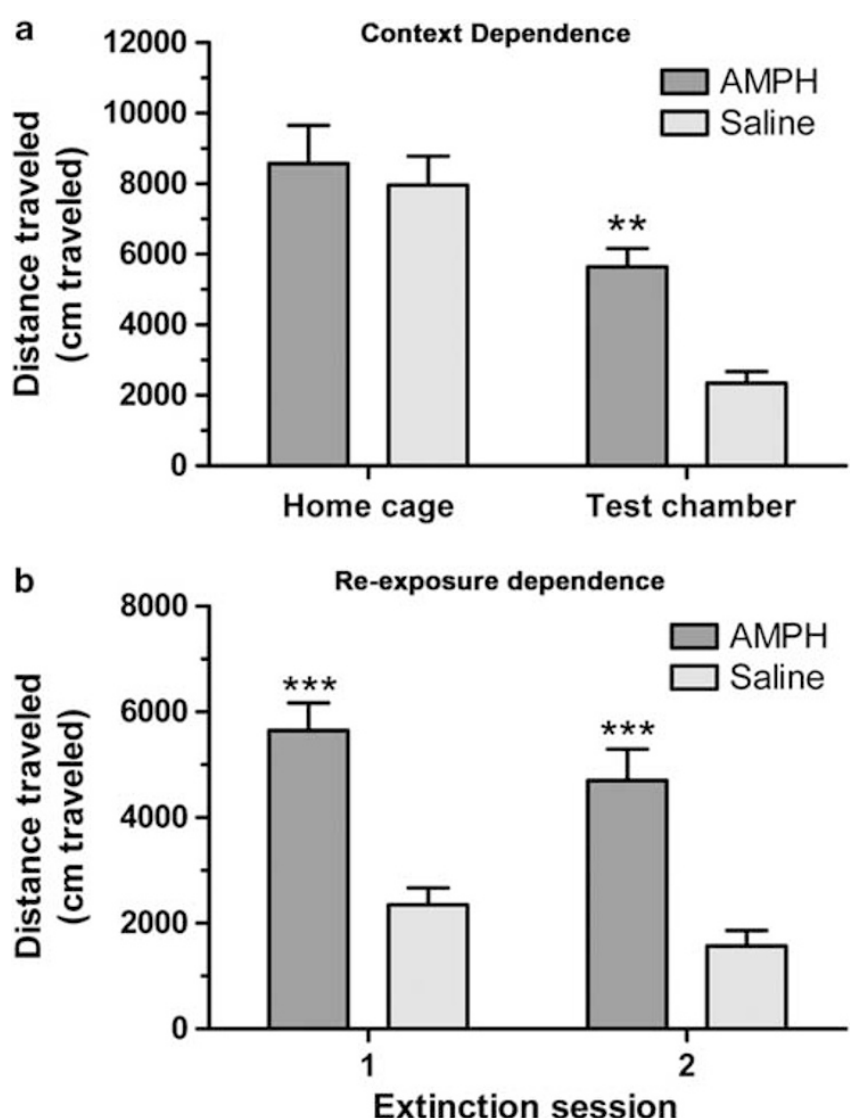

Figure 2 Characteristics of conditioned hyperactivity in WT mice. WT mice ( $n=10$ per group) were sensitized to AMPH, as previously described, in order to produce conditioned hyperactivity (observed following saline injection). In order to demonstrate the context dependency of the condition hyperactivity mice were tested in the paired test chamber or a home cage-like environment as shown in (a). AMPH-sensitized mice are hyperactive (as compared with saline pre-exposed) only in the paired test chamber (** $p<0.01$, Bonferroni). In order to test that the decline in conditioned hyperactivity was extinction, mice were re-exposed to the paired test chamber in a second extinction session 10 days after the first test as shown in (b). AMPH-sensitized mice display similar conditioned hyperactivity in both tests (**** $<0.001$, Bonferroni). Results are presented as means $\pm \mathrm{SEM}$.

$p<0.001$, Bonferroni). Two-way ANOVA confirmed significant main effects of session $(\mathrm{F}(1,18)=7.76, p<0.05)$ and AMPH treatment $(\mathrm{F}(1,18)=33.2, p<0.001)$, but no interaction of the two $(\mathrm{F}(1,18)=0.07)$.

\section{Acquisition and Expression of Behavioral Sensitization} in SR $-I-$ and GlyT1- $I+$ Mice

We used SR and GlyT1 mutant mouse strains to investigate the role of altered NMDA receptor signaling in the acquisition and expression of behavioral sensitization. SR $-I-$ mice and WT littermates were injected with either AMPH $(3.0 \mathrm{mg} / \mathrm{kg})$ or saline ( $n=23-28 /$ group $)$ immediately before activity monitoring daily for five consecutive days (Figure 3a). Both genotypes show a progressive increase in the sensitivity to AMPH over the 5 days, but the SR-Imice acquire sensitization more slowly and to a lesser extent. Two-way repeated measures ANOVA of the AMPHtreated subjects showed main effects of test day $(\mathrm{F}(4,216)=53.8, p<0.001)$ and genotype $(\mathrm{F}(1,216)=4.71$, 

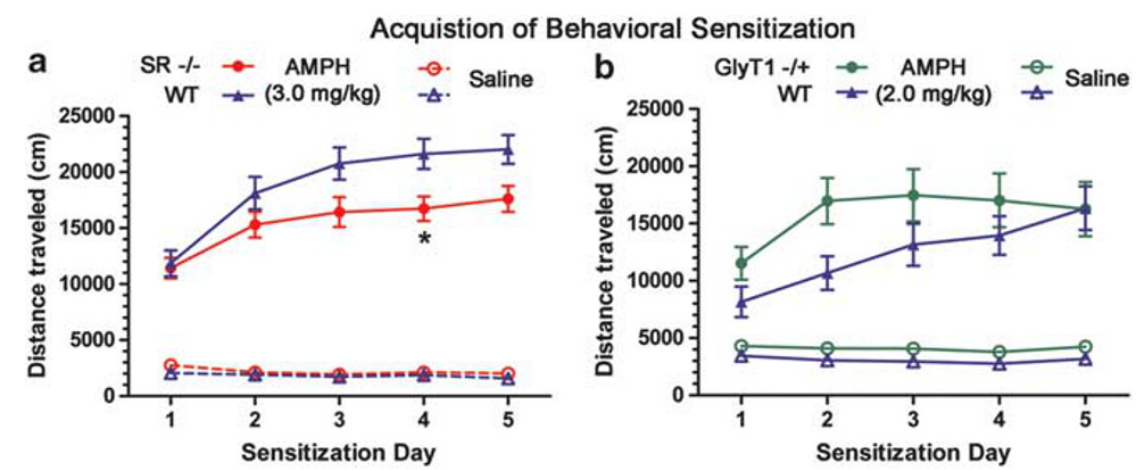

Expression of Behavioral Sensitization
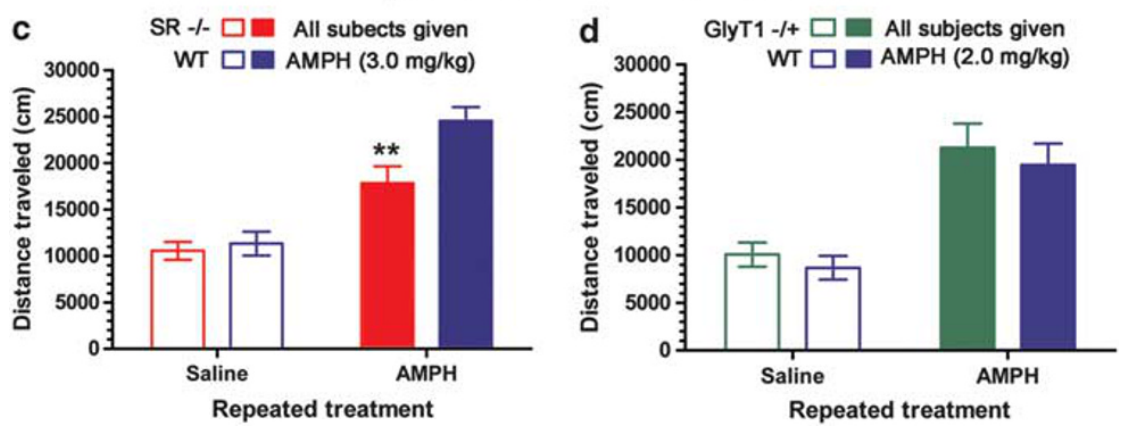

Figure 3 Acquisition and expression of context-specific behavioral sensitization in SR-/- and GlyTI-I+ mice. SR-/- and WT littermate mice $(n=23-28)$ were exposed to AMPH $(3.0 \mathrm{mg} / \mathrm{kg}$, closed symbols) or saline (open symbols) for 5 days before activity monitoring. (a) The acquisition of sensitization, with SR-1- showing a reduced AMPH response as compared with WT control (* $<<0.05$, Bonferroni). (c) The expression of sensitization in a challenge test (AMPH $3.0 \mathrm{mg} / \mathrm{kg}$ to all subjects). SR-/- again show a reduced sensitized response as compared with WT controls (** $<<0.01$, Bonferroni). GlyTI - I + and WT littermate mice ( $n=12-13)$ were exposed to AMPH (2.0 mg/ $/ \mathrm{kg}$, closed symbols) or saline (open symbols) for 5 days before activity monitoring. (b) The acquisition of sensitization, with GlyTI - / + showing a hastened acquisition, although no difference between genotypes was observed at any single time point. (d) The expression of sensitization in a challenge test, where no difference between GlyTI - I + and WT was observed. Results are presented as means \pm SEM.

$p<0.05)$ and an interaction of the two $(\mathrm{F}(4,216)=4.07$, $p<0.01)$. The post hoc analysis showed that SR $-/-$ mice were specifically less sensitive to AMPH than were WT controls on day 4 ( $p<0.05$, Bonferroni). The expression of sensitization was assessed 14 days later following an injection of AMPH $(3.0 \mathrm{mg} / \mathrm{kg})$. The data demonstrate that the reduced sensitization in SR-I- mice persists through the drug-free period (Figure 3c). Two-way ANOVA found significant main effects of the sensitizing AMPH treatment $(\mathrm{F}(1,70)=46.7, p<0.001)$ and genotype $(\mathrm{F}(1,70)=6.21$, $p<0.05)$ and an interaction of the two $(\mathrm{F}(1,70)=3.89$, $p<0.05)$. The post hoc analysis showed a statistically significant $27 \%$ reduction in AMPH efficacy in SR-Isubjects, compared with WT, sensitized to AMPH ( $p<0.01$, Bonferroni).

The effect of increased NMDA receptor signaling on the development of behavioral sensitization was examined using GlyT1-I+ subjects. GlyT1-I+ and WT subjects were injected with either AMPH $(2.0 \mathrm{mg} / \mathrm{kg})$ or saline $(n=$ 12-13/group) immediately before $60 \mathrm{~min}$ of activity monitoring daily for five consecutive days (Figure $3 \mathrm{~b}$ ). The lowered dose of AMPH was used to avoid a ceiling effect on AMPH efficacy. WT subjects showed a progressive increase in the sensitivity to AMPH. However, the GlyT1-I+ subjects became fully sensitized and reached a plateau response after a single AMPH injection. Repeated measures two-way ANOVA showed a main effect of test day
$(\mathrm{F}(4,92)=25.5, p<0.001)$ and an interaction with genotype $(\mathrm{F}(1,92)=5.15, p<0.001)$. The post hoc analysis did not identify a difference in AMPH efficacy between the genotypes on any single day. Subsequently, GlyT1-/+ and WT subjects showed similar behavior in the test for the expression of sensitization (Figure 3d). Consistent with this, a two-way ANOVA showed only a significant main effect of previous AMPH exposure $(\mathrm{F}(1,48)=33.0, p<0.001)$.

\section{Extinction of Conditioned Hyperactivity in SR-I- and GlyT1 $-I+$ Subjects}

The extinction of conditioned hyperactivity in the SR-Iand GlyT1-/ + strains was tested in order to study the role of altered NMDA receptor signaling on the long-term stability of drug-free behavior associated with chronic drug intake. SR $-1-$ and WT littermate mice sensitized to AMPH and saline-treated controls were re-exposed to the test chamber following a saline injection ( $n=15-16 /$ group $)$ in 10 extinction sessions (Figure 4). Sensitized SR-I- mice displayed a modest increase in activity as compared with the saline controls that were resistant to extinction (Figure 4a). Repeated measures two-way ANOVA found main effects of sensitizing AMPH exposure $(F(1,270)=$ $8.01, p<0.01)$ and extinction day $(\mathrm{F}(9,270)=6.68$, $p<0.001)$. The WT littermates displayed a much more robust conditioned hyperactivity that showed extensive 

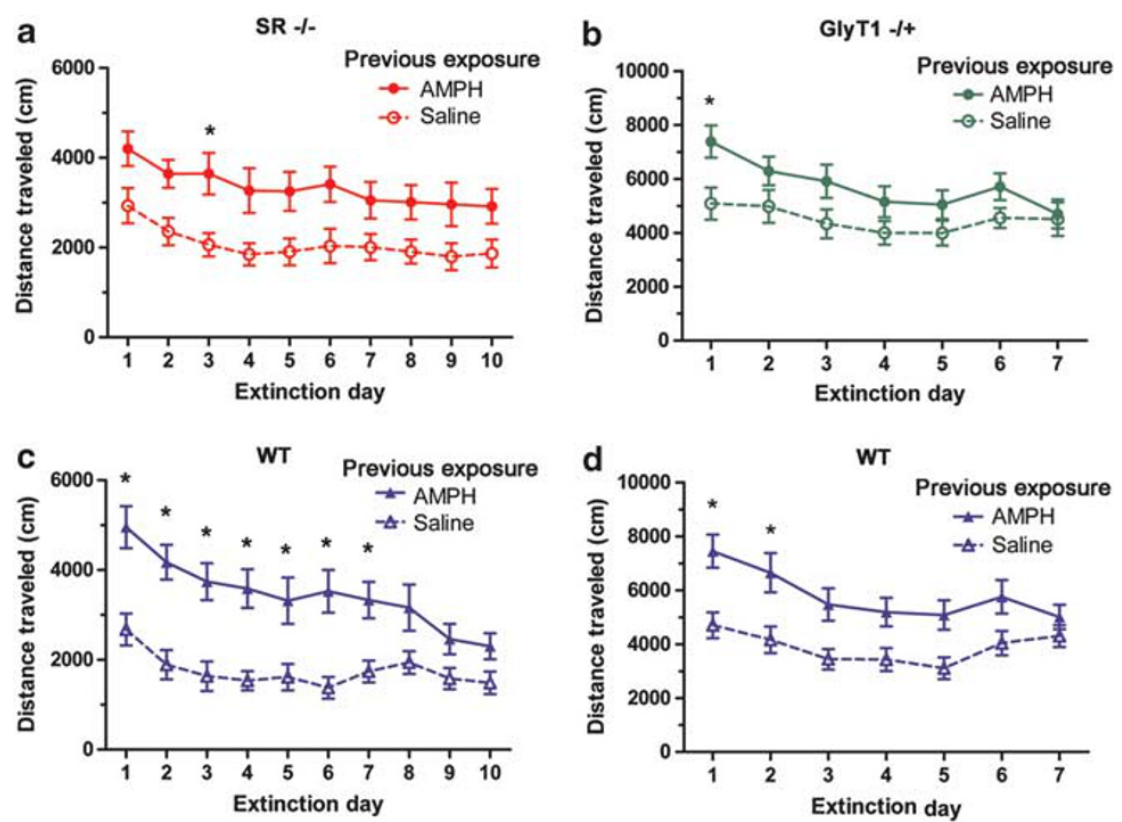

Figure 4 Extinction of conditioned hyperactivity in SR-/- and Gly TI - / + mice. Extinction of conditioned hyperactivity in SR-/- and WT littermate mice $(n=15-16)$ is shown in (a) and (b) respectively. Both genotypes express conditioned hyperactivity, comparison of AMPH and saline pre-exposure, but only WT littermate show a prominent extinction. Extinction of conditioned hyperactivity in GlyTI - I + and WT littermate mice ( $n=12-13)$ is shown in (b) and (d) respectively. Both genotypes express conditioned hyperactivity and a similar pattern of extinction. *Significant hyperactivity as compared with same genotype saline control ( $p<0.05$, Bonferroni). Results are presented as means \pm SEM.

reduction through extinction training (Figure 4c). Repeated measures two-way ANOVA showed a significant main effects of sensitizing AMPH exposure $(\mathrm{F}(1,261)=16.19, p<0.001)$ and extinction day $(\mathrm{F}(9,261)=13.72, p<0.001)$ with a significant interaction of the two terms $(\mathrm{F}(9,261)=3.83$, $p<0.001)$. In order to make genotype comparisons, conditioned hyperactivity was expressed as a difference score between sensitized and nonsensitized groups. Repeated measures two-way ANOVA showed a significant main effect of extinction training $(\mathrm{F}(9,261)=4.62, p<0.001)$ and an interaction of extinction and genotype $(\mathrm{F}(9,261)=2.00$, $p<0.05$ ).

A similar analysis of the extinction of conditioned hyperactivity was performed using GlyT1-I+ and WT littermates $(n=12-13)$. Extinction of conditioned hyperactivity was analyzed for 7 days because of the hastened extinction in the GlyT1-/+ subjects (Figure 4). GlyT1-/+ mice displayed a modest conditioned hyperactivity that fully extinguished by day 7 (Figure $4 \mathrm{~b}$ ). Repeated measures twoway ANOVA showed a main effect of extinction $(\mathrm{F}(6,138)=8.40, p<0.001)$, a trend main effect of sensitizing AMPH exposure $(\mathrm{F}(1,138)=3.67, p=0.068)$, and a trend interaction of extinction day and AMPH exposure $(\mathrm{F}(6,138)=2.04, p=0.064)$. WT subjects showed a more robust conditioned hyperactivity that also extinguished by day 7 (Figure 4d). Repeated measures two-way ANOVA showed significant main effects of extinction day $(\mathrm{F}(6,132)=9.05, p<0.001)$ and sensitizing AMPH exposure $(\mathrm{F}(1,132)=10.32, p<0.01)$, and a trend interaction of extinction day and AMPH exposure $(\mathrm{F}(6,132)=2.02$, $p=0.067)$. Genotype comparison suggested that the difference between the two groups lies in the extent of conditioned hyperactivity, not the rate of extinction, as two-way ANOVA showed only a significant main effect of genotype
$(\mathrm{F}(1,161)=4.74, p<0.05)$. It is also noteworthy that all subjects in this study seemed to have an elevated baseline activity as compared with the previous experiment with the $\mathrm{SR}-I-$ subjects. Saline-treated controls in this experiment maintained a locomotor response of $\sim 4000 \mathrm{~cm}$, as compared with $\sim 2000 \mathrm{~cm}$ in the former (SR-I-) experiment.

\section{Experience-Dependent Changes in NAcc Spine Density in SR-I- and WT Mice}

The extinction of conditioned hyperactivity was used in the present study to determine whether the structural changes are correlated with conditioned hyperactivity or the sensitized drug response (which persists after extinction of hyperactivity). The SR $-/-$ mice are useful to this end as well, as they express reduced sensitization but extinction-resistant conditioned hyperactivity. Dendritic spine density was determined in SR $-I-$ and WT mice from three experimental conditions ( $n=11-14 /$ condition): control, sensitized, and extinguished. SR $-/-$ and WT littermates displayed similar spine densities in the control condition and comparable increases following sensitization, but diverged after extinction training, with the SR-Iremaining elevated and the WT littermates returning toward the control level (Figure 5). The effect of sensitization and extinction were separately analyzed. Two-way ANOVA of the control and sensitized conditions showed a significant main effect of sensitization $(\mathrm{F}(1,45)=23.1$, $p<0.001)$. There was no effect of genotype, and post hoc analysis showed both groups were significantly elevated from control $(p<0.01$, Bonferroni). Two-way ANOVA of sensitized and extinguished conditions showed a significant interaction of extinction training and genotype $(\mathrm{F}(1,43)=4.51, p<0.05)$, with post hoc analysis confirming 


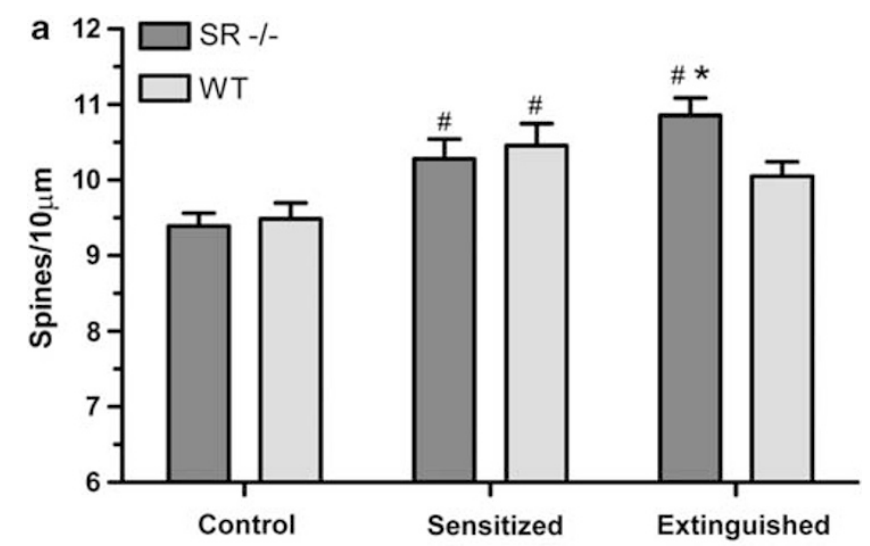

b

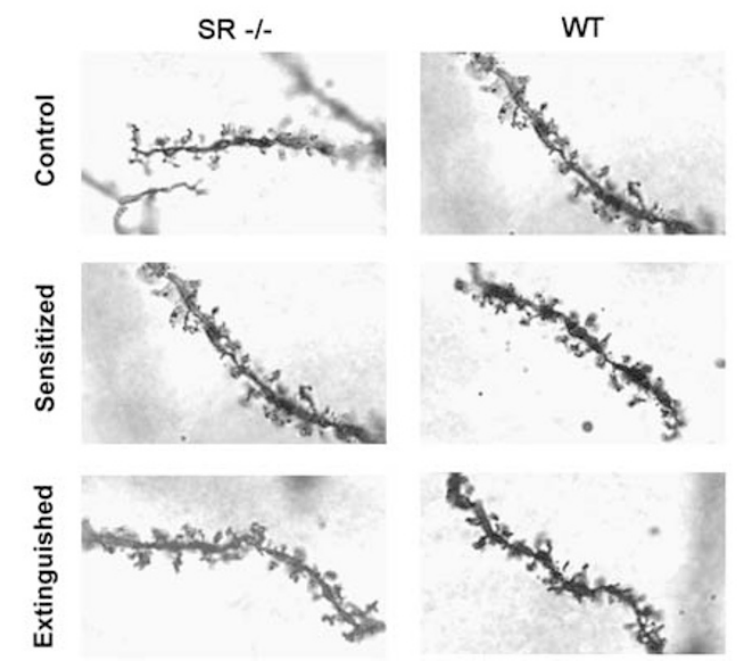

Figure 5 Experience-dependent changes in NAcc spine density in SR-I- and WT mice. (a) The analysis of nucleus accumbens core (NAcc) spine density in Golgi-fixed sections of SR-I- and WT littermate mice following repeated saline exposure (control), repeated AMPH exposure (sensitized), or following extinction of conditioned hyperactivity in AMPHexposed (extinguished) subjects. "Significant difference as compared with the control condition of the same genotype $(p<0.0 \mathrm{I}$, Bonferroni). *Significant difference between genotypes of the same condition ( $p<0.05$, Bonferroni). Results are presented as means \pm SEM. (b) Examples of Golgi-stained terminal dendrites for each condition and genotype are shown.

a difference between $\mathrm{SR}-/-$ and WT after extinction training $(p<0.05$, Bonferroni).

\section{DISCUSSION}

The present findings provide evidence supporting the role for NMDA receptor-mediated neurotransmission in extinction of conditioned behavior and neurostructural changes in the mesolimbic dopamine system driven by drugassociated stimuli. Using a context-paired sensitization paradigm the present studies investigated two behaviors, one elicited by re-exposure to drug (sensitization) and the other elicited by drug-associated stimuli in the absence of drug (conditioned hyperactivity). Reduced GMS activation in the SR $-I-$ strain caused a reduction in the degree of conditioned hyperactivity that was expressed (on extinction day 1), but a resistance of that hyperactivity to be extinguished by repeated exposure to the conditioned stimulus (test chamber). This persistent conditioned hyperactivity in the SR-I- subjects supports the conclusion that intact NMDA receptor signaling is critical to extinction learning. This genetic evidence builds upon pharmacological findings with augmented GMS activation (Paolone et al, 2009; Thanos et al, 2009; Myers and Carlezon, 2010) in models of drug-associated conditioning and suggests that NMDA receptor hypofunction may be a pathophysiological feature that increases susceptibility to addiction by inhibiting the extinction of conditioned behavior. Acquisition of behavioral sensitization was also affected by altered GMS activation, with the GlyT1-/+ subjects showing a hastened rate of acquisition and the SR $-/$ - subjects showing a delayed rate. The GlyT1-/+ subjects quickly reached a plateau response to AMPH, which remained stable during drug withdrawal. This stands in contrast to the SR $-I-$ subjects, who demonstrated a reduced level of sensitization, which persisted after drug withdrawal.

Perhaps the most interesting finding of this work is the NMDA receptor activity-dependent reversal of the AMPHinduced increase in NAc spine density that accompanied the extinction of conditioned hyperactivity. Previous research had shown that a sensitizing psychostimulant regimen results in an increase in the spine density and dendritic complexity in the NAc (Robinson and Kolb, 1997, 1999). Increased spine density develops independently of the route of administration, as self-administered cocaine also increases spine density (Robinson et al, 2001). Recent findings suggest that increased spine density is the result of associative learning. Context-paired AMPH sensitization leads to conditioned hyperactivity and an increase in NAc spine density, whereas AMPH injected daily into the ventral tegmental area produces a sensitized response without the concomitant conditioned hyperactivity or spine density changes (Singer et al, 2009). Home cage-administered cocaine, which does not lead to a conditioned response, also does not produce lasting changes in spine density (Shen et al, 2009). The effects of extinction training in the present studies demonstrate that the spine density changes reflect neuronal alterations that direct the expression of conditioned hyperactivity rather than behavioral sensitization. This is a more direct line of evidence of the linkage between NAc spine density changes and the expression of conditioned responses than shown in previous studies. Furthermore, like conditioned hyperactivity, the extinction-induced reversal of spine density changes is dependent upon intact NMDA receptor signaling, as the SR $-I-$ subject displays a failure to show extinction-related spine density reduction. This extinction-resistant spine plasticity in the NAc of the SR $-I-$ subject builds on observations of reduced dendritic complexity and spine density in the cortex of both constitutive and conditional SR mutants to point a role for NMDA receptor signaling to the bidirectional regulation of dendritic spines in multiple brain regions (Devito et al, 2010; Balu et al, 2012; Balu and Coyle, 2012).

The dissociation of behavioral sensitization and conditioned hyperactivity suggests that these are distinct behaviors that may have separable, yet interacting, neurocircuitry controlling their expression and maintenance. Behavioral sensitization is enhanced and made context dependent by pairing drug exposure with distinct environmental cues 
(Stewart and Vezina, 1991; Anagnostaras and Robinson, 1996; Mattson et al, 2008). The ability of that conditioned stimulus (drug-paired context) to produce a conditioned response (hyperactivity) on its own and still control the response to the unconditioned stimulus (drug exposure) after extinction of the conditioned response is consistent with theories that environment can serve as both an excitatory conditioned stimulus and an occasion setter (Vezina and Leyton, 2009). The degree to which these actions are separable and are regulated by independent neurocircuitry is open to speculation, but the demonstration that the spine density increases in the NAc are reversed by extinction suggests that this site may selectively regulate the excitatory conditioned stimulus properties. This role for the NAc to preferentially encode the conditioned response is further supported by the distribution patterns of SR and GlyT1 and the relative effect that the mutants had on conditioned hyperactivity. The reduced expression and extinction of conditioned hyperactivity in the SR-Isubjects would then be consistent with the prominent NAc expression of SR, whereas GlyT1 expression is significantly lower in this region (Zafra et al, 1995; Basu et al, 2009). Questions still remain as to the expression of contextspecific sensitization after extinction in the SR and GlyT1 mutants. Such studies would provide further insight in to the neurobiological distinctions between the associative roles of context, specifically whether NMDA receptor signaling is differentially involved.

The results demonstrate that NMDA receptor signaling is involved in the neurobiological processes that control the extinction of conditioned responses to drug-associated stimuli. Exposure to drug-associated stimuli is an important factor in relapse to drug seeking in addicts (O'Brien et al, 1992; Everitt et al, 2001). In this way, addiction can be seen as a disease of pathological associative learning. NMDA receptors play a well-established role in the form of synaptic plasticity thought to underlie learning and memory, making them important targets in the delineation of disease pathophysiology and treatment (Coyle, 2006; Myers et al, 2011). Recent research has demonstrated that inhibiting presynaptic glutamate inhibitory autoreceptors, and thus augmenting glutamatergic synaptic neurotransmission, reduced craving in cocaine-addicted patients (Amen et al, 2011). Preclinical work with the GMS partial agonist Dcycloserine demonstrates that the selective potentiation of NMDA receptor signaling may be an effective means of enhancing extinction of drug conditioning (Paolone et al, 2009; Thanos et al, 2009; Myers and Carlezon, 2010), in a manner similar to the extinction of conditioned fear in agoraphobic patients (Ressler et al, 2004).

Finally, these results may speak to the high prevalence of substance abuse among those suffering from schizophrenia. Pharmacologic, post-mortem, and genetic findings provide convincing evidence that NMDA receptor hypofunction is a core feature of schizophrenia (Coyle et al, 2010). The SR-Imouse strain shares behavioral and neuropathological features with schizophrenia (Basu et al, 2009; Devito et al, 2010; Balu et al, 2012). Given the inability of the SR-/- mice to extinguish AMPH-induced conditioned hyperactivity, perhaps the NMDA receptor hypofunction in schizophrenia contributes to the high prevalence of substance abuse by impairing their ability to remain abstinent (Coyle, 2006).

\section{ACKNOWLEDGEMENTS}

We thank Dr William Carlezon and Dr Ole Isacson for the generous use of their equipment and software. We also thank Dr William Carlezon, Dr Glenn Konopaske, Dr Darrick Balu, and Dr Mark Thomas for their helpful discussions, as well as Jiamin Feng for the genotyping and maintenance of our mouse colony. This work was supported by an Andrew P. Merrill Research Fellowship granted to $\mathrm{MAB}$, and National Institutes of Health grants R01MH05190 and P50MH0G0450 to JTC.

\section{DISCLOSURE}

Dr Joseph T. Coyle has received consulting fees from Abbott Pharmaceuticals, Bristol-Myers Squibb, Eli Lilly, Puretech Ventures, and EnVivo Pharmaceuticals. He holds a patent on the use of D-serine for the treatment of serious mental illness; the patent is owned by the Massachusetts General Hospital but could yield royalties if developed. Dr Michael Benneyworth declares no conflict of interest.

\section{REFERENCES}

Agatsuma S, Dang MT, Li Y, Hiroi N (2010). N-methyl-D-aspartic acid receptors on striatal neurons are essential for cocaine cue reactivity in mice. Biol Psychiatry 67: 778-780.

Amen SL, Piacentine LB, Ahmad ME, Li SJ, Mantsch JR, Risinger $\mathrm{RC}$ et al (2011). Repeated $\mathrm{N}$-acetyl cysteine reduces cocaine seeking in rodents and craving in cocaine-dependent humans. Neuropsychopharmacology 36: 871-878.

Anagnostaras SG, Robinson TE (1996). Sensitization to the psychomotor stimulant effects of amphetamine: modulation by associative learning. Behav Neurosci 110: 1397-1414.

Badiani A, Browman KE, Robinson TE (1995). Influence of novel versus home environments on sensitization to the psychomotor stimulant effects of cocaine and amphetamine. Brain Res 674: 291-298.

Balu DT, Basu AC, Corradi JP, Cacace AM, Coyle JT (2012). The NMDA receptor co-agonists, d-serine and glycine, regulate neuronal dendritic architecture in the somatosensory cortex. Neurobiol Dis 45: 671-682.

Balu DT, Coyle JT (2012). Neuronal d-serine regulates dendritic architecture in the somatosensory cortex. Neurosci Lett 517: 77-81.

Basu AC, Tsai GE, Ma CL, Ehmsen JT, Mustafa AK, Han L et al (2009). Targeted disruption of serine racemase affects glutamatergic neurotransmission and behavior. Mol Psychiatry 14: 719-727.

Bear MF, Malenka RC (1994). Synaptic plasticity: LTP and LTD. Curr Opin Neurobiol 4: 389-399.

Coyle JT (2006). Substance use disorders and Schizophrenia: a question of shared glutamatergic mechanisms. Neurotox Res 10: 221-233.

Coyle JT, Balu D, Benneyworth M, Basu A, Roseman A (2010). Beyond the dopamine receptor: novel therapeutic targets for treating schizophrenia. Dialogues Clin Neurosci 12: 359-382.

Devito LM, Balu DT, Kanter BR, Lykken C, Basu AC, Coyle JT et al (2010). Serine racemase deletion disrupts memory for order and alters cortical dendritic morphology. Genes Brain Behav 10: $210-222$.

Everitt BJ, Dickinson A, Robbins TW (2001). The neuropsychological basis of addictive behaviour. Brain Res Brain Res Rev 36: $129-138$. 
Haracz JL, Belanger SA, MacDonall JS, Sircar R (1995). Antagonist of N-methyl-D-aspartate receptors partially prevent the development of cocaine sensitization. Life Sci 57: 2347-2357.

Heusner CL, Palmiter RD (2005). Expression of mutant NMDA receptors in dopamine D1 receptor-containing cells prevents cocaine sensitization and decreases cocaine preference. J Neurosci 25: 6651-6657.

Kim HS, Park WK, Jang CG, Oh S (1996). Inhibition by MK-801 of cocaine-induced sensitization, conditioned place preference, and dopamine-receptor supersensitivity in mice. Brain Res Bull 40: 201-207.

Koya E, Golden SA, Harvey BK, Guez-Barber DH, Berkow A, Simmons DE et al (2009). Targeted disruption of cocaineactivated nucleus accumbens neurons prevents context-specific sensitization. Nat Neurosci 12: 1069-1073.

Li Y, Wolf ME (1999). Can the "state-dependency" hypothesis explain prevention of amphetamine sensitization in rats by NMDA receptor antagonists? Psychopharmacology (Berl) 141: 351-361.

Malenka RC, Nicoll RA (1999). Long-term potentiation-a decade of progress? Science 285: 1870-1874.

Martina M, ME BT, Halman S, Tsai G, Tiberi M, Coyle JT et al (2005). Reduced glycine transporter type 1 expression leads to major changes in glutamatergic neurotransmission of CA1 hippocampal neurones in mice. J Physiol 563: 777-793.

Mattson BJ, Koya E, Simmons DE, Mitchell TB, Berkow A, Crombag HS et al (2008). Context-specific sensitization of cocaine-induced locomotor activity and associated neuronal ensembles in rat nucleus accumbens. Eur J Neurosci 27: 202-212.

Myers KM, Carlezon Jr WA (2010). D-cycloserine facilitates extinction of naloxone-induced conditioned place aversion in morphine-dependent rats. Biol Psychiatry 67: 85-87.

Myers KM, Carlezon Jr WA, Davis M (2011). Glutamate receptors in extinction and extinction-based therapies for psychiatric illness. Neuropsychopharmacology 36: 274-293.

O’Brien CP, Childress AR, McLellan AT, Ehrman R (1992). Classical conditioning in drug-dependent humans. Ann NY Acad Sci 654: 400-415.

Paolone G, Botreau F, Stewart J (2009). The facilitative effects of Dcycloserine on extinction of a cocaine-induced conditioned place preference can be long lasting and resistant to reinstatement. Psychopharmacology (Berl) 202: 403-409.
Ressler KJ, Rothbaum BO, Tannenbaum L, Anderson P, Graap K, Zimand E et al (2004). Cognitive enhancers as adjuncts to psychotherapy: use of $\mathrm{D}$-cycloserine in phobic individuals to facilitate extinction of fear. Arch Gen Psychiatry 61: 1136-1144.

Robinson TE, Gorny G, Mitton E, Kolb B (2001). Cocaine selfadministration alters the morphology of dendrites and dendritic spines in the nucleus accumbens and neocortex. Synapse 39: 257-266.

Robinson TE, Kolb B (1997). Persistent structural modifications in nucleus accumbens and prefrontal cortex neurons produced by previous experience with amphetamine. J Neurosci 17: 8491-8497.

Robinson TE, Kolb B (1999). Alterations in the morphology of dendrites and dendritic spines in the nucleus accumbens and prefrontal cortex following repeated treatment with amphetamine or cocaine. Eur J Neurosci 11: 1598-1604.

Shen HW, Toda S, Moussawi K, Bouknight A, Zahm DS, Kalivas PW (2009). Altered dendritic spine plasticity in cocainewithdrawn rats. J Neurosci 29: 2876-2884.

Singer BF, Tanabe LM, Gorny G, Jake-Matthews C, Li Y, Kolb B et al (2009). Amphetamine-induced changes in dendritic morphology in rat forebrain correspond to associative drug conditioning rather than nonassociative drug sensitization. Biol Psychiatry 65: 835-840.

Stewart J, Vezina P (1991). Extinction procedures abolish conditioned stimulus control but spare sensitized responding to amphetamine. Behav Pharmacol 2: 65-71.

Thanos PK, Bermeo C, Wang GJ, Volkow ND (2009). D-cycloserine accelerates the extinction of cocaine-induced conditioned place preference in C57bL/c mice. Behav Brain Res 199: 345-349.

Tsai G, Ralph-Williams RJ, Martina M, Bergeron R, BergerSweeney J, Dunham KS et al (2004). Gene knockout of glycine transporter 1: characterization of the behavioral phenotype. Proc Natl Acad Sci USA 101: 8485-8490.

Tsien JZ (2000). Linking Hebb's coincidence-detection to memory formation. Curr Opin Neurobiol 10: 266-273.

Vezina P, Leyton M (2009). Conditioned cues and the expression of stimulant sensitization in animals and humans. Neuropharmacology 56(Suppl 1): 160-168.

Zafra F, Aragon C, Olivares L, Danbolt NC, Gimenez C, StormMathisen J (1995). Glycine transporters are differentially expressed among CNS cells. J Neurosci 15: 3952-3969. 